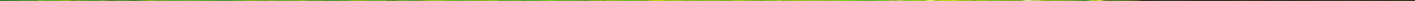




\section{Las Trayectorias Reproductivas en el Contexto Familiar en la Ciudad de Manzanillo, Provincia del Granma, Cuba}

Lorenzo I. Herrera LeÓn*

RESUMEN. El presente trabajo pretende relacionar las condiciones socioeconómicas de vida familiar con las conductas reproductivas de mujeres insertadas en los hogares. Factores como el color de la piel del jefe de hogar, su ocupación, su escolaridad, la relación de parentesco de la mujer con él, entre otros, integran un conjunto de elementos explicativos de importancia. Las diferencias en las condiciones socioeconómicas de vida familiar podrían también poner de relieve diferencias de género así como en los conocimientos sobre salud reproductiva entre las mujeres. Otro factor que puede aportar explicaciones en las conductas de las mujeres más jóvenes es el número de parejas estables e hijos tenidos con otras relaciones por sus progenitores, lo que podría ser una tentativa para aquilatar las brechas intergeneracionales en este aspecto. El trabajo se basa en un estudio de casos de mujeres jóvenes residentes en la ciudad de Manzanillo, provincia de Granma, Cuba. El criterio de selección consistió en tomar a aquellas embarazadas que acudieron a las consultas programadas de obstetricia entre marzo y junio del año 2011. Se usan dos instrumentos para captar la información, uno referido a las historias reproductivas y el otro para las condiciones socioeconómicas del hogar.

Palabras claves: conductas reproductivas, brechas intergeneracionales, condiciones socioeconómicas de vida familiar, género, salud sexual y reproductiva.

ABSTRACT. This work pretends to make a relation between the families' social economic life conditions with the reproductive behavior of women inserted at homes. Facts as the head of household color skin, occupation, studies, parental relationship whit the woman who he lives, and more, it makes some importance explicative elements. The different in the families' social economic life conditions could be put outside the gender differences and also the knowledge about reproductive health between women. Another fact that can give explications about younger women behavior is the number of stable couples and parents children had in other relationships, what could be a tentative to value the intergenerational gap in this aspect. The work is based in a study of resident younger women case in the city of Manzanillo, Province of Granma, Cuba. The selection judgment was base in took those pregnant women that were in programmed obstetric consults between March and June of 2011. Two instruments were use to capture information, one referred the reproductive history and other for the homes social economic conditions.

Keywords: Reproductive behavior, intergenerational gap, families' life social economic conditions, gender, sexual and reproductive health.

\section{Introducción}

La trayectoria reproductiva se ha definido como la sucesión de eventos vitales de la historia reproductiva que ha experimentado la mujer (embarazos, interrupciones, pérdidas intrauterinas, nacimientos, muertes infantiles), que incluye además la intensidad y espaciamiento temporal de esos eventos y los episodios de morbilidad acaecidos a la gestante y al producto de la concepción. Dicha tra- yectoria tiene sin lugar a dudas un estrecho vínculo con tradiciones y patrones culturales y familiares heredados y presumiblemente con las condiciones socioeconómicas de vida de la familia.

Estas condiciones de vida en el seno hogareño están estrechamente vinculadas con las condiciones materiales de la vivienda (infraestructura, servicios básicos, otros servicios), el nivel de ingreso familiar, los niveles educacionales y culturales adquiridos por sus miembros. A lo

Recibido: enero de 2012 /Aceptado y versión final julio de 2012.

* Dr. en Demografía, Centro de Estudios Demográficos, Universidad de La Habana

e-mail: lorenzo@cedem.uh.cu 
anterior se suma la cuestión del caudal de conocimientos en educación para la salud y particularmente en cuanto a salud sexual (SS) y salud reproductiva (SR), que incluye todo lo referente a los aspectos higiénicos, fisiológicos y psicológicos que propician una mejor selección por parte de la pareja o de la mujer del momento idóneo para la reproducción, cuidado, espaciamiento y número de su descendencia; en este punto el conocimiento y uso eficiente de métodos anticonceptivos es un elemento crucial.

Importantes investigaciones han confirmado la estrecha relación entre las condiciones socioeconómicas del hogar y la fecundidad, y muy especialmente han advertido notables diferencias tanto en los patrones como en los niveles de la maternidad adolescente según diferencias en las condiciones de vida familiar (Flórez, 2005: 389-391), (Flórez y Soto, 2006: 5-26). Es de suponer entonces que diferencias en las condiciones socioeconómicas de vida familiar puedan establecer diferencias en la conformación de estrategias y proyectos individuales de vida de las mujeres y de las parejas, lo que podría a su vez incidir en las trayectorias reproductivas y muy particularmente en la intensidad y espaciamiento de los eventos que la conforman.

No menos importante es tener en cuenta el color de la piel, factor que hasta donde ha sido dable conocer, ha adolecido de poca presencia en estudios anteriores de índole reproductiva en nuestro país pero que ha ido adquiriendo cada vez más un notable protagonismo por su alto poder explicativo, y en ese sentido puede propiciar variaciones importantes tanto en las condiciones socioeconómicas del hogar como en las trayectorias reproductivas femeninas.

En el plano reproductivo la provincia Granma ${ }^{1}$ presenta una alta prevalencia de interrupciones de embarazos (abortos y regulaciones menstruales) muy superior a la media nacional (interrupciones por mil mujeres entre 12 y 49 años: Granma: 61.3 vs. Cuba: 56.9), (Gran, 2005), y hoy en día exhibe un número promedio de hijos por mujer que está entre los cuatro más elevados de la nación
(TGF: Granma 1.69 vs. Cuba 1.59). Además, Granma se encuentra entre las primeras cuatro provincias con mayor índice de maternidad adolescente del país (Tasa de fecundidad de menores de 20 años: Granma 62.2 vs. Cuba 50.1) (Minsap, 2008), que aún considerando los bajos niveles de fecundidad de la nación, su peso relativo es el tercero más alto, acumulando las adolescentes de ese territorio más del $18 \%$ del promedio de hijos por mujer, superada solamente por las provincias de Holguín y Las Tunas (Minsap, 2008).

\section{Metodología}

El presente estudio es en esencia de índole exploratoria y de esta esencia dimana su sentido diagnóstico.

El núcleo del estudio reside en evaluar la asociación entre dos tipologías construidas de manera independiente una con respecto a la otra, la primera de condiciones socioeconómicas de vida familiar y la otra de trayectorias reproductivas. Ambas tienen como base el uso del sistema Análisis de conglomerado en dos etapas (Two Step Cluster Analysis). En el diseño de ambas se procuró no introducir variables que establecieran a priori, una asociación o correlación por construcción, a modo de hacer a cada tipología lo más pura posible en términos estadísticos, con el propósito de que si existiera asociación entre ambas, se debiera a una relación inherente entre nivel de vida familiar y la reproducción.

\subsection{Objetivos de la Investigación}

\section{General}

- Caracterizar las trayectorias reproductivas femeninas en su relación con las condiciones socioeconómicas de vida familiar.

\section{Específicos}

- Caracterizar los hogares donde se insertan las mujeres involucradas en el estudio según las condiciones socioeconómicas de vida familiar.

1. Una de las 15 provincias de Cuba. Surge a raíz de la última división político administrativa aprobada en agosto de 2010, y asume su nombre en 1976 en honor al desembarco en playa Las Coloradas del Granma, punto de desembarque del Che Guevara y Fidel Castro con otros guerrilleros. 
- Caracterizar las trayectorias reproductivas femeninas de las mujeres involucradas en el estudio.

- Verificar correspondencia entre las trayectorias reproductivas femeninas y las condiciones de vida familiar de los hogares donde se insertan las mujeres involucradas en el estudio.

- Valorar conocimientos sobre algunos elementos y prácticas en SS y SR en las mujeres involucradas en el estudio.

- Valorar diferencias intergeneracionales en aspectos de la reproducción (de progenitores a hijas)

\subsection{Hipótesis de Trabajo}

Diferencias en las condiciones socioeconómicas de vida familiar entre hogares propician diferencias en las trayectorias reproductivas femeninas. Las mujeres de hogares con nivel socioeconómico más elevado muestran trayectorias reproductivas menos abultadas (menor número de embarazos, de nacidos vivos, de interrupciones, etc.) y una actitud más positiva con respecto a SS y SR (edades más propicias para la tenencia de hijos, mayor prevalencia anticonceptiva, mayores conocimientos sobre nutrición al bebé, menor propensión a hábitos tóxicos, y en general proyectos familiares mejor estructurados) que otras insertadas en hogares con menor nivel en las condiciones socioeconómicas de vida familiar.

\subsection{Descripción de la Muestra}

La muestra para esta investigación consistió en la selección de 137 hogares donde residen mujeres embarazadas que asisten a las consultas regulares programadas de ginecología y obstetricia de cinco áreas de salud del municipio Manzanillo, provincia de Granma. La información fue recogida entre el mes de marzo y hasta la primera quincena de junio del año 2011 por personal de salud altamente calificado.

El procedimiento utilizado consistió en entrevistar a la mujer embarazada luego de concluida su consulta con el especialista en obstetricia y ginecología y previa solicitud a ella y lectura de los propósitos de la investigación y consentimiento informado, por conducto del cual decidía voluntariamente someterse al interrogatorio. Los hogares seleccionados se escogieron a través de la mujer entrevistada, la que respondió las preguntas de ambos instrumentos.

\subsection{Tipos de Familia}

Referente a los tipos de familias, el predominante es el nuclear con una presencia de 50\%, seguida del extendido, compuesto con algo más del 48\%. El primero de estos porcentajes se aproxima al dado para la totalidad del país por Benítez (Benítez, 2009).

Otras características adicionales son por ejemplo, la existencia de una sola familia unipersonal que corresponde a una mujer con un hijo que no vive con ella. En casi las $3 / 4$ partes de los hogares el jefe vive con su pareja y en los casos en que no es así por lo general la jerarquía corresponde a personas del sexo femenino. Hay pocas familias con presencia de tres generaciones contadas desde el jefe de hogar hasta los nietos y muy pocas con cuatro generaciones convivientes, desde el jefe de hogar hasta el bisnieto (Anexo 1).

\subsection{Tipología de Hogares}

La construcción de una tipología de objetos se basa en la similitud en cuanto a las propiedades y características de los mismos. En el caso de los hogares estos pueden considerarse objetos que presentan una serie de características individuales que de acuerdo a la similitud de las mismas podrían agregarse en diferentes grupos para cada uno de los cuales la discrepancia entre sus miembros se espera sea la mínima.

El procedimiento para detectar tipologías de hogares que se ha utilizado es el Análisis de cluster en dos etapas (TwoStep Cluster Analysis).

Para la construcción de una tipología de hogares en cuanto a aspectos de índole socioeconómica, se tomó una serie de variables que por su construcción no implicara algún compromiso con la historia reproductiva de las mujeres, en el sentido de evitar la llamada asociación o correlación incorporada (In-built correlation). Por tanto, el conjunto de variables debe buscar desde las cuestiones 
sociales y económicas, hasta la calidad de la vivienda y el grado de confort con que viven las familias.

Las variables que fueron seleccionadas para la conformación de la clasificación de los hogares:

- Jefe del hogar: edad, sexo, calificación, ocupación, color de piel, estado civil.

- Mujer embarazada (encuestada): nivel de escolaridad, ocupación, parentesco con respecto al jefe de hogar.

- Vivienda: tipo de vivienda, índice de carga económica, índice de hacinamiento, local cocina (común, exclusivo, vía de abasto de agua), baño (común, exclusivo o no, vía de abasto de agua), servicio sanitario (exclusivo o no, tipo, vía de abasto de agua), fuente de abasto agua, frecuencia de abasto de agua, desagüe, equipos electrodomésticos básicos (radio, radiograbadora con o sin CD, TV en colores, TV en blanco y negro, plancha eléctrica, refrigerador, lavadora, máquina de coser, ventilador, cocina eléctrica, licuadora, batidora, olla arrocera, olla reina, bicicleta, teléfono de línea), equipos complementarios (aire acondicionado, video, DVD, computadora, auto, camión, tractor, moto, ciclomotor, teléfono celular, afectaciones de la vivienda techo, paredes, apuntalamiento).

De la aplicación del procedimiento descrito las familias fueron clasificadas en dos grupos, cluster 1 y cluster 2, que de ahora en adelante se denominarán CSE I y CSE II en alusión a las Condiciones Socio Económicas. La representación esencialmente gráfica del procedimiento ilustra las cualidades de cada uno, lo que desplaza la balanza a favor del CSE I como aquel cuyos hogares poseen las mejores condiciones socioeconómicas (Cuadro No.1).

Cuadro No. 1. Ciudad de Manzanillo; Granma: Clasificación de los Hogares Según las Condiciones SocioEconómicas

\begin{tabular}{|l|c|l|}
\multicolumn{1}{|c|}{ Clúster } & No. de Hogares & Nivel Socioeconómico \\
\hline CSE I & 97 & Mayor que en CSE II \\
\hline CSE II & 35 & Menor que en CSE I \\
\hline No clasificado & 5 & \\
\hline Total & 137 & \\
\hline
\end{tabular}

Fuente: elaboración propia, 2011

\subsection{Tipología de Trayectorias Reproductivas}

De manera similar a la ocasión en que se estableció una tipología de hogares desde el punto de vista socioeconómico, ahora corresponde esta tarea para las trayectorias reproductivas de las mujeres embarazadas que fueron encuestadas.

El procedimiento para ello es el mismo utilizado en aquella ocasión, el Análisis de conglomerados en dos etapas (TwoStep Clusters Analysis).

Las variables seleccionadas a fin de clasificar a las mujeres constituyen puntos nodales en el sentido de que marcan momentos de relevancia en la secuencia de eventos de sus vidas reproductivas. Al mismo tiempo poseen alto poder descriptivo y discriminatorio en el sentido estadístico.

Las variables incluidas fueron las siguientes: edad actual, edad menarquía, edad primera relación sexual, tipo de relación afectiva a la primera relación sexual (pareja estable, relación casual), si quedó o no embarazada en la primera relación sexual, en qué terminó el embarazo de la primera relación sexual (en caso de haber quedado embarazada), embarazos previos, regulaciones menstruales, abortos espontáneos, abortos provocados, abortos farmacológicos, embarazos múltiples, total de nacidos vivos tenidos, estado civil actual, episodios de morbilidad del embarazo (sepsis vaginal, sepsis urinaria, ganancia insuficiente de peso, anemia, bajo peso, hipertensión del embarazo, asma, diabetes gestacional, cardiopatías, malformaciones congénitas).

De este ejercicio se obtuvo una tipología de trayectorias reproductivas en dos grupos, cluster 1 y cluster 2 , nombrados en esta ocasión TREP I y TREP II en alusión a trayectoria reproductiva. Ambas denominaciones se utilizarán indistintamente en el discurso. Solamente una mujer resultó no clasificada al presentar un valor faltante en alguna variable de las consideradas, razón por la cual el procedimiento la descarta (Cuadro No.2). 
Cuadro No. 2. Ciudad de Manzanillo; Granma: Trayectorias Reproductivas

\begin{tabular}{|l|l|c|}
\multicolumn{1}{|c|}{ Clúster } & \multicolumn{1}{|c|}{ Característica } & Total \\
\hline TREP I & Más abultada que TREP II & 76 \\
\hline TREP II & Menos abultada que TREP I & 60 \\
\hline No clasificado & & 1 \\
\hline Total & & 137 \\
\hline
\end{tabular}

Fuente: elaboración propia, 2011

\section{Condiciones Socioeconómicas de Hogares y Trayectorias Reproductivas}

Para establecer la correspondencia entre las condiciones socioeconómicas de hogares y las trayectorias reproductivas se realizó el cruzamiento de la clasificación de los hogares en los cuales se insertan las mujeres entrevistadas y la clasificación de lo que se ha denominado sus trayectorias reproductivas de forma simultánea.

Esta manera de enfocar la correspondencia entre condiciones socioeconómicas de vida familiar y reproducción permite cruzar las dos tipologías, construidas de forma independiente, de manera que en una tabla de doble entrada se sitúen las mujeres en los diferentes escaques.

$\mathrm{Al}$ cruzar los dos criterios de clasificación, atendiendo a las condiciones socioeconómicas del hogar y a los tipos de trayectorias reproductivas, las 137 mujeres encuestadas quedaron dispuestas como se observa en el Cuadro No.3.

El total de mujeres se redujo debido a que no clasificaron 6 mujeres, 5 en CSE y 1 en la TREP.
La valoración de la asociación entre las dos clasificaciones (hogares y trayectorias reproductivas) se pudo constatar a través del uso del test estadístico Ji- cuadrado.

$\mathrm{Al}$ valor Ji-cuadrado obtenido de 0,61 con 1 grado de libertad le corresponde un p-valor igual a 0,435 por lo que la relación se declara no significativa desde el punto de vista estadístico. Es decir, no existe una evidencia fundamentada para rechazar la idea de la independencia entre las dos clasificaciones.

En otras palabras, las distribuciones de las mujeres según la variable Tipo de Trayectoria Reproductiva no difieren significativamente entre CSE (58\%-42\% en CSE I y 50\%-50\% en CSE II) y las diferencias que se observan deben ser atribuidas a la participación del azar. Este acontecimiento refuta la hipótesis planteada inicialmente la cual asevera que existe una correspondencia inversa entre el nivel socioeconómico del hogar y el tipo de trayectoria reproductiva de las mujeres insertadas en los mismos. De manera más clara, la evidencia empírica dice que el $71 \%$ de los hogares clasifican en el grupo de mayor nivel socioeconómico (CSE I) y de estos el 58\% (sus mujeres en estudio) se encuentran en el grupo de trayectorias más abultadas, el TREP I. Al mismo tiempo, aquellos hogares procedentes del nivel socioeconómico más bajo (CSE II) comparten mitad por mitad, su presencia en ambos tipos de trayectorias. El 56\% de todas las mujeres encuestadas clasifican en el tipo de trayectoria reproductiva más abultada o TREP I, lo cual hace de esta la predominante.

\section{Cuadro No. 3. Ciudad de Manzanillo; Granma: Condiciones Socioeconómicas de Vida en el Hogar y Clasificación de las Trayectorias Reproductivas Femeninas}

\begin{tabular}{|c|c|c|c|c|}
\hline \multirow{2}{*}{\multicolumn{2}{|c|}{$\begin{array}{l}\text { Condiciones socioeconómicas } \\
\text { de vida en el hogar }\end{array}$}} & \multicolumn{3}{|c|}{ Clasificación de trayectorias reproductivas femeninas } \\
\hline & & \multirow{2}{*}{$\begin{array}{c}\text { Más abultada que en II } \\
\text { TREP I }\end{array}$} & \multirow{2}{*}{$\begin{array}{c}\text { Menos abultada que en I } \\
\text { TREP II }\end{array}$} & \multirow[b]{2}{*}{ Total } \\
\hline Clasificación de hogares & Nivel socioeconómico & & & \\
\hline CSE I & Mayor que en 2 & $\begin{array}{c}56 \\
(58 \%)\end{array}$ & $\begin{array}{c}41 \\
(42 \%)\end{array}$ & $\begin{array}{c}97 \\
(100 \%)\end{array}$ \\
\hline CSE II & Menor que en 1 & $\begin{array}{c}17 \\
(50 \%) \\
\end{array}$ & $\begin{array}{c}17 \\
(50 \%)\end{array}$ & $\begin{array}{c}34 \\
(100 \%)\end{array}$ \\
\hline Total & Promedio & $\begin{array}{c}73 \\
(56 \%)\end{array}$ & $\begin{array}{c}58 \\
(44 \%)\end{array}$ & $\begin{array}{c}131 \\
(100 \%)\end{array}$ \\
\hline
\end{tabular}

Fuente: elaboración propia, 2011 
Al margen de lo comentado en los párrafos precedentes, un elemento importante a tomar en cuenta es que las mujeres ubicadas en TREP I tienen una edad promedio alrededor de 3 años superior a la de TREP II (27 vs. 24) y esto hace más probable que tengan una historia reproductiva con más acumulación de eventos. La misma situación se evidencia dentro de cada CSE, la tendencia de las mujeres mayores a agruparse en la TREP I y las más jóvenes en TREP II (Cuadro No.4).

Cuadro No. 4. Edad Promedio de las Mujeres Según Nivel Socioeconómico de Hogares y Tipo de Trayectoria Reproductiva

\begin{tabular}{|l|c|c|c|}
\hline CSE I & TREP I & TREP II & Total \\
& 26 & 23 & 25 \\
\hline CSE II & $29 \%)$ & $(42 \%)$ & $(100 \%)$ \\
& $(50 \%)$ & 25 & 27 \\
\hline Total & 27 & 24 & $(100 \%)$ \\
& $(56 \%)$ & $(44 \%)$ & 26 \\
& $(100 \%)$ \\
\hline
\end{tabular}

Fuente: elaboración propia, 2011

Con el propósito de controlar el efecto confusor de la variable edad se realizó una post estratificación en tres grupos de edades, constatándose que la relación entre status socioeconómico familiar y el tipo de trayectoria no guardan asociación alguna, toda vez que los tests Ji- Cuadrado practicados no resultaron significativos en ninguno de los tres estratos (Cuadro No.5).

Cuadro No. 5. Prueba de Ji Cuadrado: Post Estratificación Relación Estatus Socioeconómica Familiar y Trayectoria Reproductiva

\begin{tabular}{|c|c|c|c|c|}
\hline & TREP I & TREP II & Total & \\
\hline CSE I & 0,42 & 0,58 & 1,00 & P30,3505 \\
\hline CSE II & 0,29 & 0,71 & 1,00 & \\
\hline & & $25-29$ & & \\
\hline & TREP I & TREP II & Total & $\mathrm{p}^{3} 0,4392$ \\
\hline CSE I & 0,71 & 0,29 & 1,00 & \\
\hline CSE II & 0,86 & 0,14 & 1,00 & \\
\hline & & $30 y+$ & & \\
\hline CSE I & 0,69 & 0,31 & 1,00 & \\
\hline CSE II & 0,71 & 0,29 & 1,00 & \\
\hline
\end{tabular}

Fuente: elaboración propia, 2011
Tomando en cuenta estos resultados parece admisible sostener la idea de que existe una independencia relativa entre las condiciones socioeconómicas de vida familiar en los hogares y las trayectorias reproductivas de las mujeres insertadas en los mismos, en el presente contexto muestral.

\subsection{Género y Condiciones Socioeconómicas del Hogar}

\subsubsection{Escolaridad}

La distribución de mujeres según el nivel de instrucción formal es bastante similar entre los diferentes status socioeconómico del hogar, solamente las del CSE I exhiben mínimas ventajas en las categorías de Preuniversitario completo e Incompleto y Secundaria Completa. Este hecho podría estar relacionado con las oportunidades de estudio en el país, que permiten el acceso universal y gratuito a cualquier esfera educativa con independencia de las condiciones de vida material de las familias (Gráfico No.1).

\subsubsection{Ocupación}

En la ocupación también existe gran similitud en la distribución de las mujeres en las distintas categorías de este factor, señalándose pequeñas diferencias que no llegan a ser de importancia. Es oportuno señalar que no se cuenta con el tipo de ocupación, es decir, el trabajo o

Gráfico No. 1. Nivel de Escolaridad de la Mujer Según Condiciones Socioeconómicas del Hogar

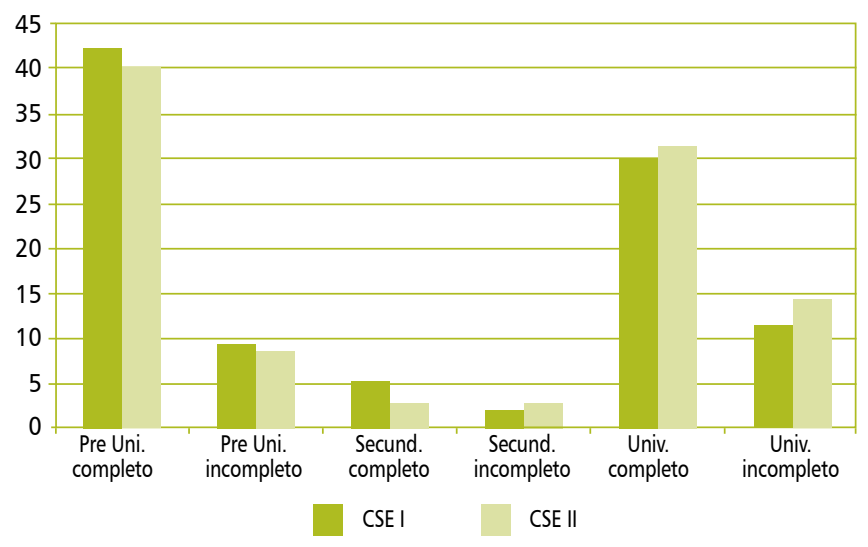

Fuente: datos de la encuesta aplicada a mujeres embarazadas en municipio Manzanillo, provincia de Granma, 2011. 
actividad que realiza la mujer, aunque es presumible que exista similitud entre ambos grupos dado el parecido entre los perfiles de escolaridad, que como se sabe, constituye un determinante de la ocupación (Gráfico No.2).

Gráfico No. 2. Ocupación de la Mujer Según Condiciones Socioeconómicas del Hogar

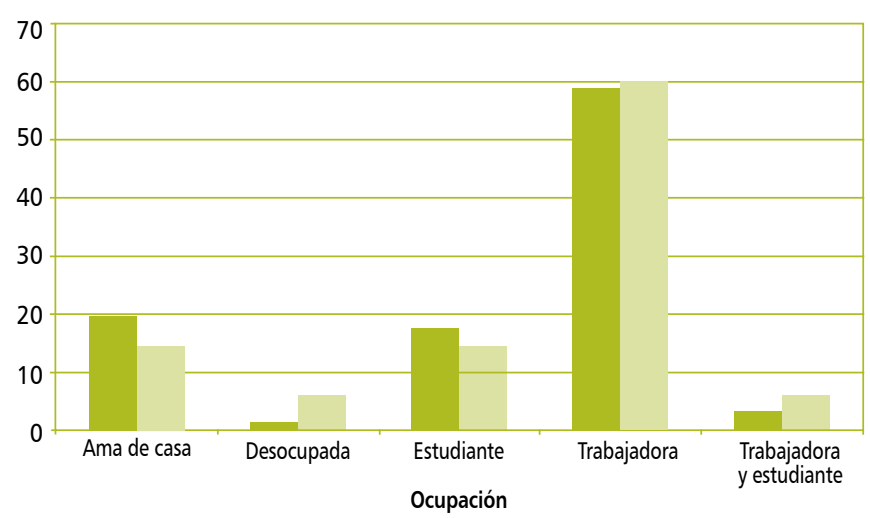

Fuente: datos de la encuesta aplicada a mujeres embarazadas en municipio Manzanillo, provincia de Granma, 2011.

\subsubsection{Jefatura del Hogar}

La pertenencia a uno u otro cluster de condiciones socioeconómicas del hogar sí establece alguna diferencia en cuanto a la jefatura del hogar. El porcentaje de mujeres del CSE I con estatus de jefa de hogar casi duplica al del CSE II. Quizás esto induzca algunas diferencias de género en el sentido de tomas de decisiones y responsabilidad en la conducción de la familia (Gráfico No.3).

Si se observa la condición de jefa de hogar en aquellas familias en las cuales está presente el esposo o compañero se observa también la superioridad en el CSE I, hecho

\section{Gráfico No. 3. Jefatura Femenina de Familia Según Condiciones Socieconómicas del Hogar}

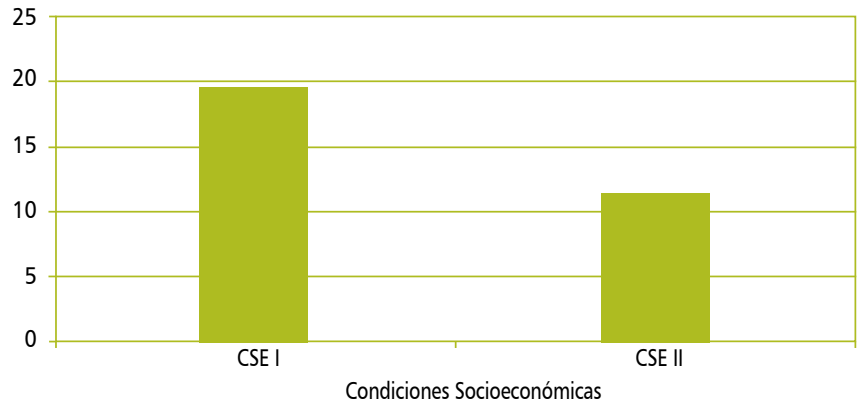

Fuente: datos de la encuesta aplicada a mujeres embarazadas en municipio Manzanillo, provincia de Granma, 2011. que confirma una especie de ruptura con la tradición de que sea por lo general el hombre el que posea la responsabilidad en la conducción de la familia (Gráfico No.4).

Gráfico No. 4. Jefatura Femenina de Familia con Presencia de Pareja, Según Condiciones Socioeconómicas del Hogar

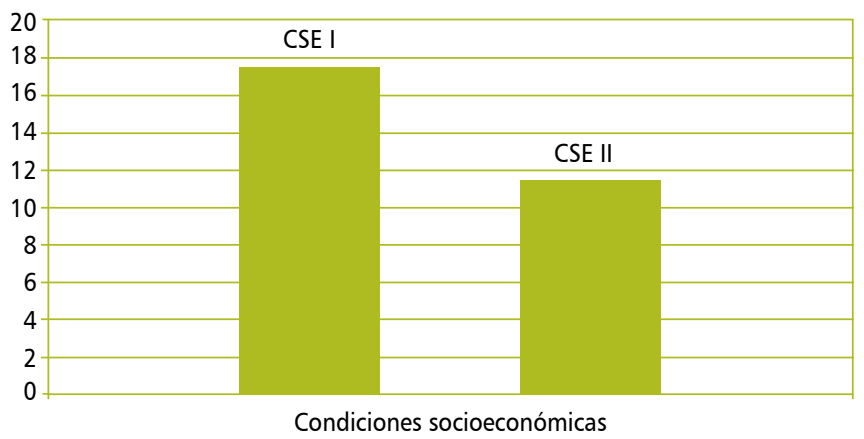

Fuente: datos de la encuesta aplicada a mujeres embarazadas en municipio Manzanillo, provincia de Granma, 2011.

\subsection{Morbilidad y Cultura en Salud Reproductiva Según} Condiciones Socioeconómicas del Hogar

\subsubsection{Maternidad Adolescente y Condiciones Socioeconó- micas del Hogar}

La maternidad en la adolescencia no es en sí misma una causa de morbilidad pero, por el alto riesgo obstétrico que representa se incluye en esta sección.

La prevalencia de maternidad en la adolescencia apunta a que algo más de la séptima parte de las mujeres encuestadas tuvieron su primer hijo con menos de 20 años. No es ocioso recordar que la provincia donde se ubica la ciudad de Manzanillo está entre las primeras con mayor maternidad en la adolescencia. La prevalencia casi se duplica para las mujeres ubicadas en el grupo de nivel socioeconómico más elevado (Cuadro No.6).

Cuadro No. 6. Maternidad Adolescente: CSE, en Porcentaje

\begin{tabular}{|l|c|}
\hline Clasificación & $\%$ \\
\hline CSE I & 16.5 \\
\hline CSE II & 8.6 \\
\hline Total* $^{*}$ & 14.4 \\
\hline
\end{tabular}

Fuente: datos de la encuesta aplicada a mujeres embarazadas en municipio Manzanillo provincia de Granma, 2011.

*Este total representa el porcentaje de mujeres que han sido madres antes de los 20 años en toda la muestra incluye el CSE I y CSE II. 


\subsubsection{Morbilidad del Embarazo y de la Familia de la Mujer} Embarazada

En cuanto a la prevalencia de los episodios de morbilidad del embarazo, se observa que no existen diferencias notables entre estratos socioeconómicos de los hogares, sobresaliendo una mayor prevalencia de Sepsis Vaginal en el CSE I y en CSE II la Anemia y Ganancia Insuficiente de Peso (Gráfico No.5). Lo mismo sucede con la morbilidad de la familia de la embarazada en la que se presentan tenues discrepancias como una mayor prevalencia de Hipertensión Arterial, seguida por Asma y Cardiopatía en CSE I, mientras que CSE II supera al

\section{Gráfico No. 5. Episodios de Morbilidad Embarazo Según Condiciones Socioeconómicas del Hogar}

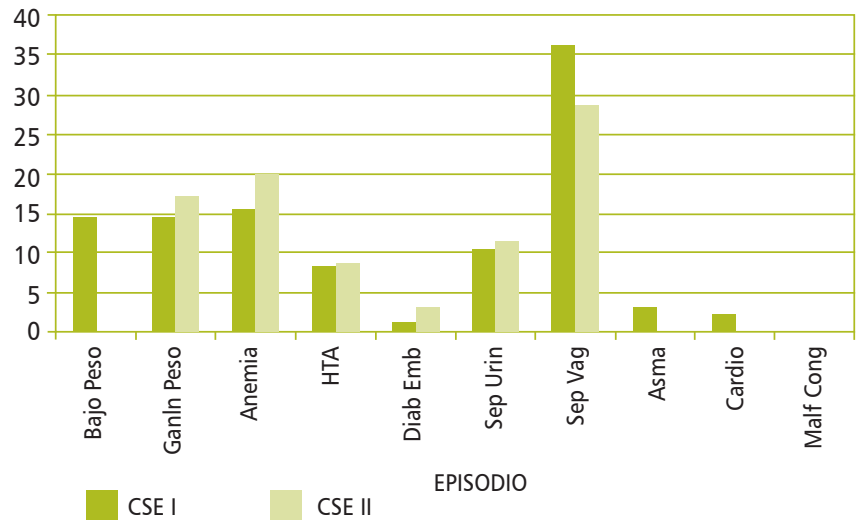

Fuente: datos de la encuesta aplicada a mujeres embarazadas en municipio Manzanillo, provincia de Granma, 2011.

\section{Gráfico No. 6. Morbilidad de la Familia Según Condicio-} nes Socioeconómicas del Hogar

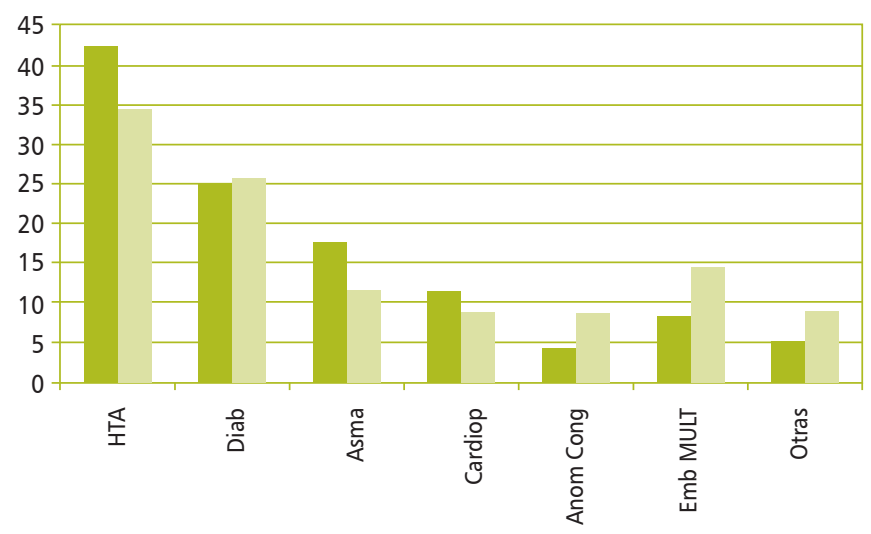

CSE I

CSE II

EPISODIO

Fuente: datos de la encuesta aplicada a mujeres embarazadas en municipio Manzanillo, provincia de Granma, 2011. primero en Anomalías Congénitas y Embarazo Múltiple, bajo el entendimiento de que este último rubro no es exactamente una enfermedad pero sí un factor de riesgo de la mortalidad perinatal (Gráfico No.6).

\subsubsection{Cultura en Salud Reproductiva y Condiciones Socioeco-} nómicas del Hogar

El conocimiento sobre los efectos del consumo de drogas por parte de la embarazada, es prácticamente el mismo en cada grupo de mujeres con predominio de casi $70 \%$ de ninguno o escaso. Esto es una alerta para las autoridades sanitarias de la localidad en materia de educación para la salud y particularmente en la salud reproductiva e induce pensar en las generaciones de mujeres jóvenes y adolescentes que aún no han comenzado su vida reproductiva y tengan carencias de estos conocimientos básicos (Gráfico No.7).

En el panorama del conocimiento sobre ciertas cuestiones de salud reproductiva, como el tiempo de espera para un nuevo embarazo después de uno que terminó en parto normal o cesárea, la edad óptima para tener los hijos, las edades de riesgo para la embarazada y el futuro bebé, los aspectos más importantes no son las diferencias entre uno y otro grupo de hogares que no son ni de mediana magnitud, sino la evidencia de una baja cultura en aspectos claves de la reproducción; sólo escapa en cierta medida a esta crítica el tiempo de lactancia, aún

Gráfico No. 7. Grado de Conocimiento Sobre Efecto de Consumo de Drogas Durante el Embarazo

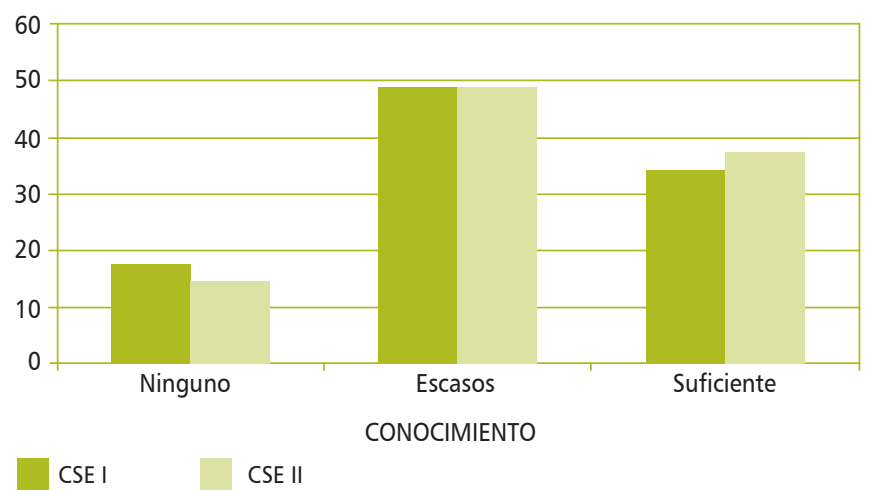

Fuente: datos de la encuesta aplicada a mujeres embarazadas en municipio Manzanillo, provincia de Granma, 2011. 
sin llenar todas las expectativas, dado que es un tema de gran divulgación popular por casi todos los medios (Gráfico No.8).

En este orden de cosas, se advierte que las causas por las que debe suspenderse la lactancia son, a grandes rasgos, del mismo grado de conocimiento por parte de las mujeres de ambos estratos y en especial el caso de padecer fiebre la madre es crítico (Gráfico No.9).

Existe similitud por la preferencia de hijos, con independencia del nivel de vida familiar y también sobre

Gráfico No. 8. Respuestas Correctas Sobre Salud Reproductiva Según Condiciones Socioeconómicas del Hogar

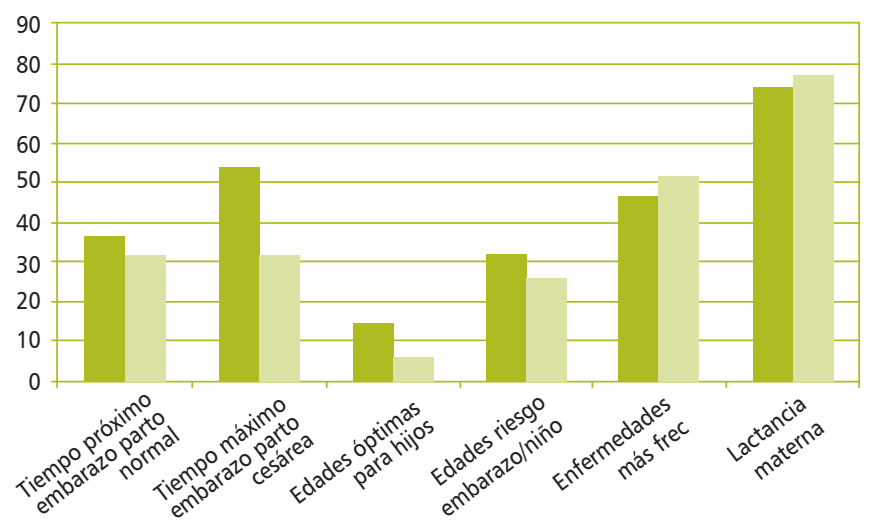

CSE I CSE II

Fuente: datos de la encuesta aplicada a mujeres embarazadas en municipio Manzanillo, provincia de Granma, 2011.

Gráfico No. 9. Respuestas Correctas Sobre Suspensión de Lactancia Materna, Según Condiciones Socieconómicas del Hogar

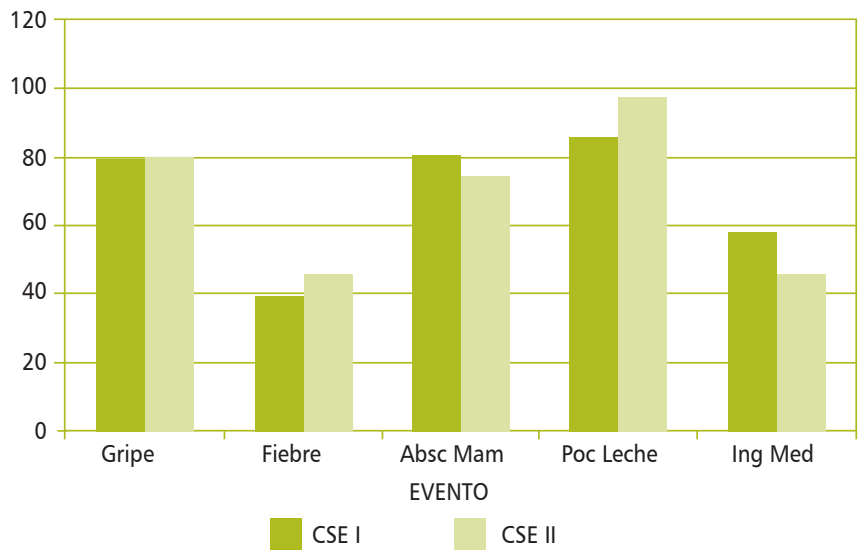

Fuente: datos de la encuesta aplicada a mujeres embarazadas en municipio Manzanillo, provincia de Granma, 2011. la percepción de riesgo de transmisión del VIH/SIDA, particularmente el conocimiento por la vía del sexo oral se dibuja como el más deficiente en todo este contexto (Gráfico No.10 y No.11).

\subsubsection{Conductas Paternas}

Las conductas paternas en cuanto a uniones y aspectos reproductivos pueden tener determinada incidencia en las conductas reproductivas de su descendencia, particularmente sobre las hijas. La edad promedio de la embarazada al tener el primer nacido vivo es ligeramente menor que la de su progenitora en algo más de tres cuartas

\section{Gráfico No. 10. Hijos Deseados Según Condiciones Socio- económicas del Hogar}

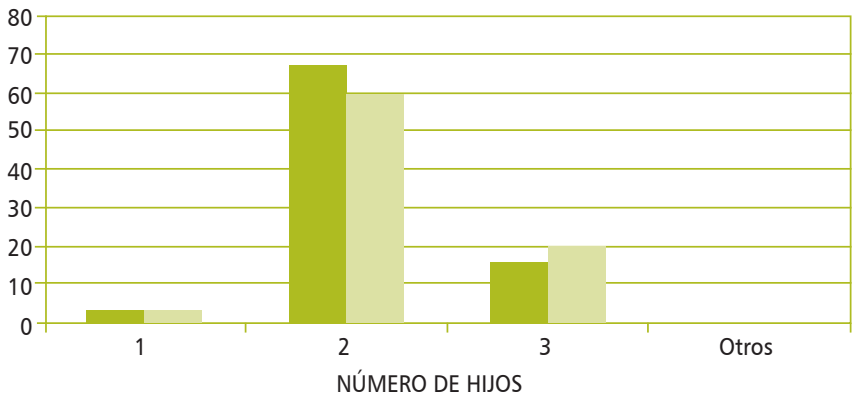

CSE I CSE II

Fuente: datos de la encuesta aplicada a mujeres embarazadas en municipio Manzanillo, provincia de Granma, 2011

Gráfico No. 11. Respuestas Correctas de Percepción de Riesgo de VIH/SIDA Según Condiciones Socieconómicas del Hogar

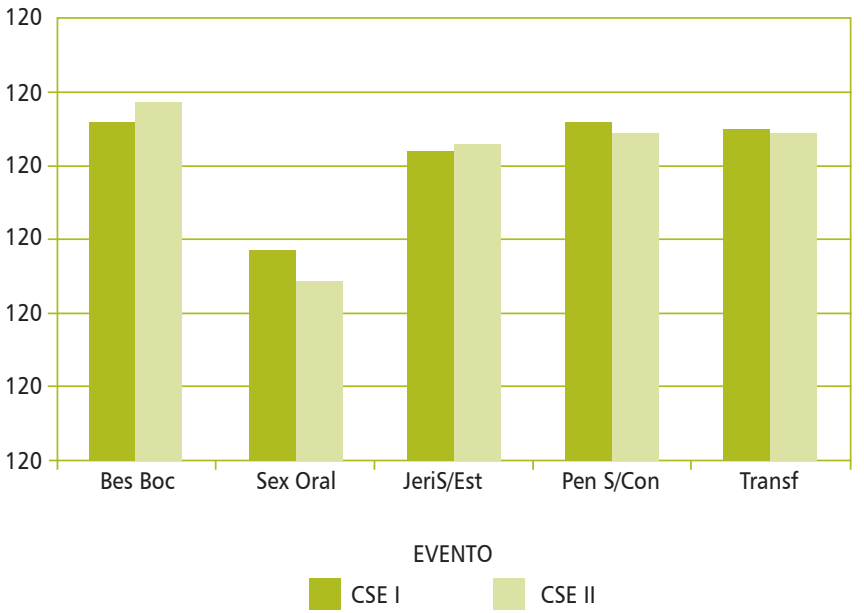

Fuente: datos de la encuesta aplicada a mujeres embarazadas en municipio Manzanillo, provincia de Granma, 2011. 
partes de año. El padre de la embarazada por su parte, tenía más de tres años y medio que la madre de aquella al momento de su nacimiento (Gráfico 12 y 13).

El nivel de instrucción formal de ambos progenitores difiere en el sentido de que las mamás poseen mayor presencia en los niveles más altos que los papás, aunque se reconoce que en esta comparación puede influir la mayor cantidad de respuesta "No Sabe" en caso de los padres. No obstante parece ser entonces que el nivel de las hijas tiene que ver más con el de la madre pues ambos patrones contienen semejanzas (Gráfico 14 y 15).

\section{Gráfico No. 12. Distribución de la Edad Padre al Tener a su Hija}

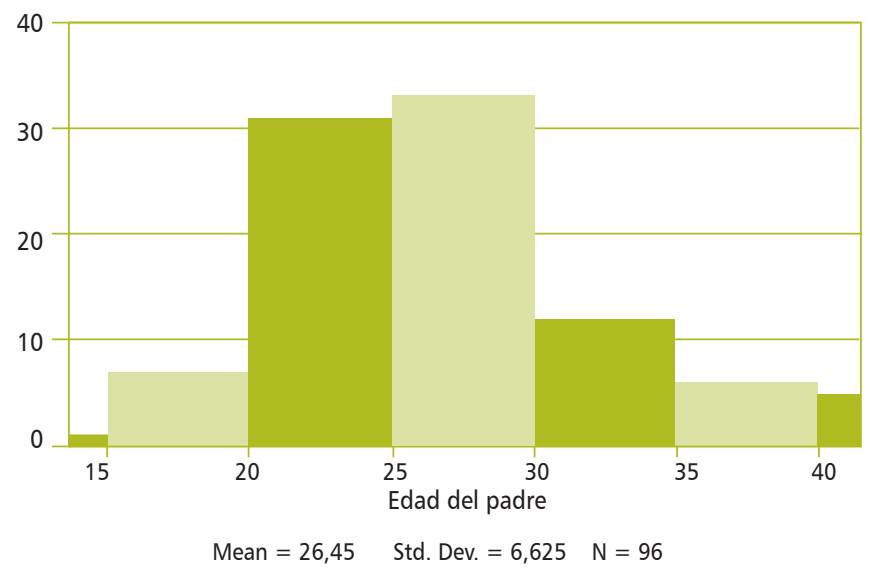

Fuente: datos de la encuesta aplicada a mujeres embarazadas en municipio Manzanillo, provincia de Granma, 2011.

Gráfico No. 13. Distribución de la Edad Madre al Tener a su Hija

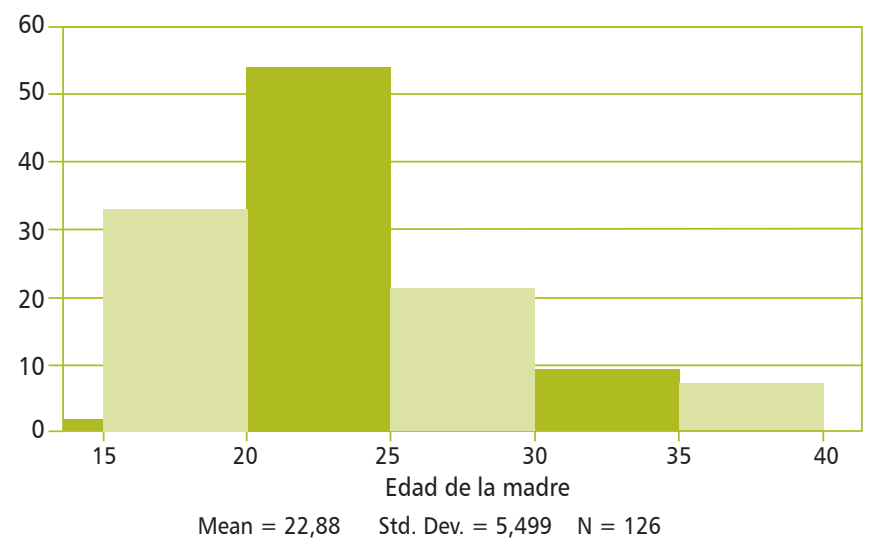

Fuente: datos de la encuesta aplicada a mujeres embarazadas en municipio Manzanillo, provincia de Granma, 2011.
Por lo general la hija embarazada sabe más sobre su mamá que sobre su papá y muchas desconocen incluso el paradero de este, razón que pudiera explicar el abultado número de mujeres hijas que declaran no saber.

Respecto a la relación de hijos tenidos por los progenitores y sus hijas descendientes, se observa que en el caso de las progenitoras la relación es bastante débil ya que las frecuencias en los perfiles- filas no muestran un nítido desplazamiento hacia órdenes superiores de naci-

\section{Gráfico No. 14. Distribución de la Escolaridad de la Madre Embarazada}

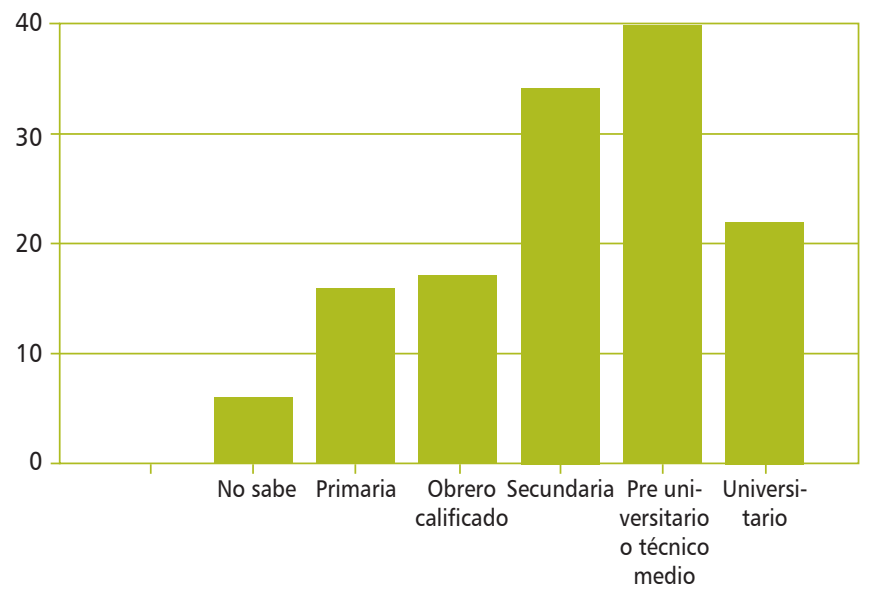

Escolaridad de la madre

Fuente: datos de la encuesta aplicada a mujeres embarazadas en municipio Manzanillo, provincia de Granma, 2011.

Gráfico No. 15 . Distribución de la Escolaridad del Padre de la Embarazada

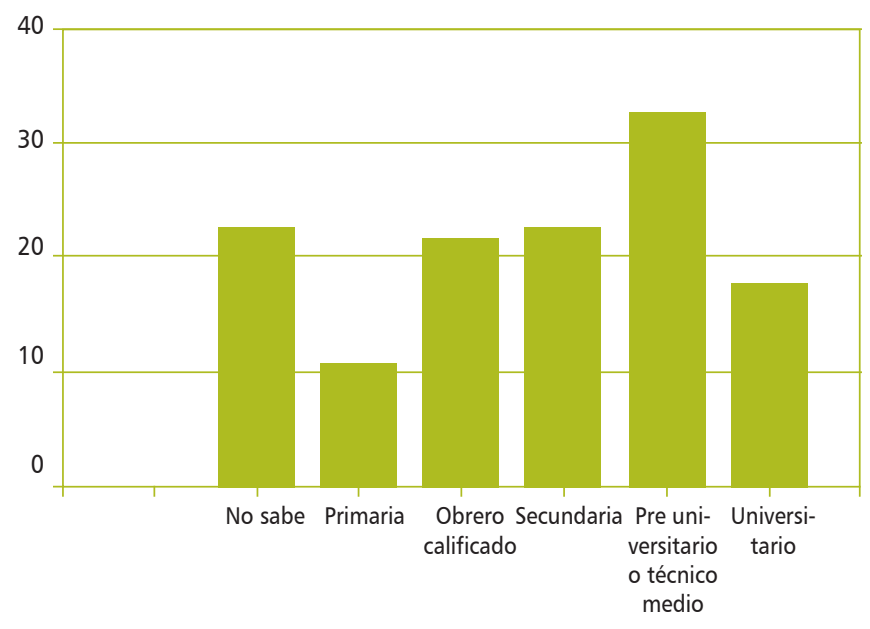

Escolaridad del padre

Fuente: datos de la encuesta aplicada a mujeres embarazadas en municipio Manzanillo, provincia de Granma, 2011. 
dos vivos de las hijas con excepción del cuarto perfil-fila $(3 \mathrm{y}+)$ para el cual se da un aumento del porcentaje de mujeres en los órdenes 1 y 2 . Respecto a los progenitores la situación es muy parecida; sólo para el cuarto perfil-fila $(3 \mathrm{y}+)$ se observa un nítido incremento en los porcentajes de mujeres ubicadas en los órdenes 1 y 2.

Un elemento importante a considerar en este análisis es que muchas de las madres jóvenes presumiblemente no han completado su vida reproductiva activa sino que más bien la han comenzado, por lo que la comparación quizás no muestra la relación que se manifestaría más claramente de haberlas concluido (Cuadro No.7).

\begin{tabular}{|c|c|c|c|c|}
\hline \multirow{2}{*}{$\begin{array}{l}\text { Otros hijos de la } \\
\text { madre }\end{array}$} & \multicolumn{3}{|c|}{ Total de Nacidos Vivos de la hija } & \multirow[b]{2}{*}{ Total } \\
\hline & 0 & 1 & 2 & \\
\hline 0 & 56,67 & 35,56 & 7,78 & 100,00 \\
\hline 1 & 60,71 & 32,14 & 7,14 & 100,00 \\
\hline 2 & 60,00 & 40,00 & 0,00 & 100,00 \\
\hline $3 y+$ & 33,33 & 55,56 & 11,11 & 100,00 \\
\hline \multicolumn{5}{|c|}{$\begin{array}{c}\text { Distribución de número de hijos de la mujer según número tenido por los } \\
\text { padres }\end{array}$} \\
\hline \multirow[b]{2}{*}{ Otros hijos del padre } & \multicolumn{3}{|c|}{ Total de Nacidos Vivos de la hija } & \\
\hline & 0 & 1 & 2 & Total \\
\hline 0 & 55,07 & 36,23 & 8,70 & 100,00 \\
\hline 1 & 54,84 & 38,71 & 6,45 & 100,00 \\
\hline 2 & 66,67 & 33,33 & 0,00 & 100,00 \\
\hline $3 y+$ & 41,67 & 50,00 & 8,33 & 100,00 \\
\hline
\end{tabular}

Fuente: elaboración propia, 2011.

\section{Consideraciones Finales}

Ante todo se impone la realización de la siguiente pregunta:

¿Por qué las mujeres de hogares con condiciones socioeconómicas mejores se concentran más en el grupo de trayectorias reproductivas en el cual existe mayor cúmulo de eventos?

Ante la tentativa de brindar algunas ideas esclarecedoras de estos comportamientos se alude por lo general al alto nivel de educación y cultura del pueblo cubano, que es un elemento de importancia suprema en relación a las conductas reproductivas pero además, debe considerarse el tipo de sistema de salud imperante que es por añadidura una garantía en estos aspectos.

El sistema de salud cubano posee ciertas características que lo hacen en cierta medida único en el planeta: universal, gratuito y accesible.

Aparte, debe señalarse que la educación sanitaria y particularmente la educación sexual y reproductiva son accesibles para todo ciudadano y ciudadana con independencia de su ingreso salarial, nivel de instrucción, procedencia, color de piel, etc. Debe añadirse que el paternalismo del sistema de salud cubano también contribuye a la eficiencia del sistema. Si el individuo no va al sistema, entonces el sistema va al individuo, esto es una clara alusión a que las autoridades de salud establecen los mecanismos para que ningún individuo quede desprotegido y si es el caso de una mujer embarazada, el sistema la capta en cualquier circunstancia.

Entonces no es de extrañar que las condiciones de vida en el seno familiar no determinen de manera absoluta los comportamientos reproductivos y existan ciertos grados de libertad entre estos y las condiciones socioeconómicas de vida en el hogar. En este mismo orden de cosas, las campañas educativas en temas concernientes a la educación sexual y reproductiva son actividades que están insertadas en el diario vivir de la sociedad cubana, y su divulgación ocupa importantes espacios radiofónicos y televisivos.

Estos elementos precedentes pueden justificar o explicar la homogeneidad que muchas veces se observa en los indicadores de las variables demográficas, a decir, en la fecundidad o la mortalidad.

No obstante pueden existir ciertas condicionantes que predispongan los resultados, por lo que es pertinente valorar algunas interrogantes:

¿Existe algún sesgo de selección que provoca esta situación? o ¿Están las mujeres de los hogares menos favorecidos implementando alguna estrategia de vida?, ¿Es una situación propia de la ciudad de Manzanillo? El hecho de comparar historias reproductivas que están en desarrollo y no han concluido podría crear algunas asimetrías comparativas. Las mujeres de mayor edad 
lógicamente tendrán mayor acumulación de eventos reproductivos que las más jóvenes y ya este hecho puede determinar en gran medida la clasificación en uno u otro tipo de trayectoria reproductiva.

La replicación de un estudio de esta índole con la inclusión de mujeres de más edad y que presumiblemente hayan concluido su vida reproductiva activa, de otras regiones o provincias del país y con aplicación de muestreo probabilístico de hogares puede contribuir a modificar, reafirmar o descartar la relación identificada en el presente estudio.

En el patrón de episodios de morbilidad del embarazo es más común entidades de tipo infeccioso como la sepsis vaginal, muy típica en las mujeres en estado de gravidez. Siguen otras que tienen que ver con el esfuerzo metabólico de la embarazada para la nutrición del feto como es la anemia del embarazo y la ganancia insuficiente de peso. En este sentido el rol familiar y la vigilancia obstétrica son de inestimable valor.

Las debilidades en cuanto a conocimientos explorados sobre salud sexual se sitúan preferentemente en aspectos relacionados con las vías de transmisión del VIH/SIDA, específicamente con la modalidad de sexo oral. Según los resultados obtenidos existe un desconocimiento bastante generalizado en este punto.

Aunque respecto a otras vías de transmisión del VIH/ SIDA (transfusión, sexo desprotegido, uso de jeringuilla sin esterilizar) existe mayor percepción del riesgo de infección, el nivel de conocimiento no alcanza lo que se denominaría el esperado, sobre todo si se toma en cuenta las diversas y perennes prácticas promocionales que se llevan a cabo en el país.

Otro punto crucial se refiere a que se ha magnificado el papel del VIH/SIDA y en cierta medida otras importantes infecciones de transmisión sexual aparecen con bajo protagonismo. Debe insistirse en este aspecto y subrayar la importancia de un cuidado integral que no sólo va dirigido al VIH/SIDA.

Respecto a la salud reproductiva existen insuficiencias de conocimientos en aspectos tales como el tiempo de espera entre embarazos sucesivos, sea después de un parto normal o una cesárea; sobre las enfermedades y padecimientos más frecuentes que pueden acontecer a la mujer embarazada; sobre las edades de riesgo para la embarazada y respecto al consumo de alcohol durante el embarazo.

La evaluación de la influencia de las conductas reproductivas paternas-y concretamente la tenencia de hijos con otras parejas-sobre las de sus hijas está limitada en el presente contexto por el escaso número de mujeres estudiadas con más de un hijo. No obstante se aprecia que cuando los progenitores presentan órdenes de nacimiento elevados (de 3 ó más hijos) sus hijas tienden a tener más hijos de orden 2 , aunque es oportuno mencionar que esa relación es más bien débil.

El primer elemento dentro del marco de una política local que refuerce la conducta sexual y reproductiva hacia una perspectiva más saludable, indudablemente debe partir de una reflexión profunda sobre el papel que juega la juventud y la adolescencia y su aporte a la reproducción. Tanto el embarazo como la maternidad adolescentes tienen importantes pesos específicos dentro de la conducta global de las mujeres estudiadas y puede ser un reflejo fiel de lo que sucede en muchas otras regiones del país. Es pertinente preguntarse si se ha hecho todo o casi todo lo posible por diseñar e implementar programas estratégicos en educación sexual y en salud reproductiva y si han estado acompañados de una táctica coherente. Si las poblaciones dianas han sido bien definidas y delimitadas y si los programas han llegado en el momento oportuno.

La masa de jóvenes y adolescentes no es una población uniforme, existen muchas veces diferencias importantes en comportamientos que pueden manifestarse en las preferencias de asociación en grupos y la conquista de sus líderes podría suponer un gran paso de avance para la diseminación de una educación sexual que convenza. Pero a la vez una estrategia en estos temas debe tener en cuenta el entorno familiar y aún más, a aquellos parientes que despierten mayor simpatía y admiración y sean una especie de ídolos para sus familiares más jóvenes. No debe olvidarse empero, el papel de la comunidad como potenciador y portador de sinergia social. 


\section{Bibliografía}

- Benítez, M. E. 2009. Dinámica de los hogares y de la familia cubanos, en Cuba: Población y Desarrollo. Centro de Estudios Demográficos, 2009. ISBN 978 959-7005-54-4.

- Courgeaux, D; Lelieve, E. (2001). Análisis Demográfico de las Biografías. Centro de Estudios Demográficos y de Desarrollo Urbano. El Colegio de México.

- Crivisqui, E. (1999). Presentación del Método de Análisis Factorial de Correspondencias Múltiples (AFCM). Presta. CD/ROM.

- Flórez, CE. (2005). Factores socioeconómicos y contextuales que determinan la actividad reproductiva de las adolescentes en Colombia. Rev Panam Salud Pública. 2005; 18(6): 388-402.

- Flórez, CE; Soto, V. (2006). Fecundidad adolescente y diferencias en Colombia y la región de América Latina y el Caribe, en Reunión de expertos sobre Población y Pobreza en América Latina y el Caribe, 14 y 15 de noviembre, 2006. CEPAL, Santiago de Chile.

- Gran, M. (2005). Interrupción voluntaria de embarazo y anticoncepción. Dos métodos de regulación de la fecundidad. Cuba 1995-2000. Dirección Nacional de Estadísticas, Ministerio de Salud Pública. ISSN: $1727-7884$.

- Gran, M. (2005). Interrupción voluntaria de embarazo y anticoncepción. Dirección Nacional de Estadísticas, Ministerio de Salud Pública. La Habana. ISSN: $1727-7884$.

- Guerrero, M. (2003). Clasificación socioeconómica de los hogares chilenos. Instituto Nacional de Estadísticas, Chile.
- Guerrero, M. (2003). Clasificación socioeconómica de los hogares chilenos. Instituto Nacional de Estadísticas, Chile.

- Leridon, H. (1977). Aspectos biométricos de la reproducción humana. Serie D. No. 1031. Celade, San José. Costa Rica.

- Ministerio de Salud Pública, Dirección Nacional de Registros Médicos y Estadísticas de Salud. (2008). Anuario estadístico de salud.

- Norusis, M. 2004. SPSS 13.0 Statistical Procedures Companion. Upper Saddle-River, N.J.: Prentice Hall, Inc.

- Ocampo, M. (2007). Estimación del índice de nivel socioeconómico 1976-2001. Informe nacional sobre desarrollo humano, documento de trabajo 3/2007. PNUD, Bolivia.

- Ocampo, M. (2007). Estimación del índice de nivel socioeconómico 1976-2001. Informe nacional sobre desarrollo humano, documento de trabajo 3-2007. PNUD, Bolivia.

\section{Anexos}

\section{Anexo 1. Características de hogares según tipo}

\begin{tabular}{|c|c|c|c|}
\hline Tipo & No. Fam. & Características & No. Fam \\
\hline Unipersonal & 1 & $\begin{array}{l}\text { Jefe hogar con presencia } \\
\text { pareja }\end{array}$ & 101 \\
\hline Monoparental & 2 & $\begin{array}{l}\text { Jefe hogar sin presencia } \\
\text { pareja }\end{array}$ & 36 \\
\hline Biparental c/hijos & 42 & Hogares con nietos & 31 \\
\hline Biparental s/hijos & 25 & Hogares con bisnietos & 8 \\
\hline \multirow[t]{2}{*}{ Sub total (Nucleares) } & 69 & Hogares s/hijos & 41 \\
\hline & & Hogares con hijo & 96 \\
\hline $\begin{array}{l}\text { Extendido/com- } \\
\text { puesto }\end{array}$ & 67 & $\begin{array}{l}\text { Jefe hogar/Nieto ( } 3 \text { gene- } \\
\text { raciones) }\end{array}$ & 9 \\
\hline Total & 137 & $\begin{array}{l}\text { Jefe hogar/bisnieto (4 } \\
\text { generaciones) }\end{array}$ & 5 \\
\hline
\end{tabular}

Fuente: elaboración propia, 2011. 